\title{
Discussion on Application of Green Building Design Based on BIM
}

\section{Technology}

\author{
(China Construction II Bureau II Construction Engineering Co., Ltd, Guangdong, Shenzhen, 518000, China)
}

\section{Meaning of BIM Technology}

BIM technology is also called the building information model. It is mainly to integrate relevant data effectively, convert all the data into the basic data, and then establish the building model and input the relevant data reasonably. In the current situation, BIM technology has 5 characteristics, namely, visibility, coordination, simulation, optimization and graphic. At present, it is very important to apply BIM technology in the management of green building design, and it is mainly to optimize project management by using the advantages of digital simulation, so as to effectively improve the construction progress, better to ensure the engineering quality and to promote the development of green building construction.

\section{Application Advantages of BIM Technology in Green Building Design}

2.1 Strong Synergy in the Design of Various Specialties

For the traditional design work, it is very easy to raise conflicts because of communication problems. BIM mainly includes topology, physics, geometry, etc. The main function of topology is to further reflect the relationship between components, while physical information mainly reflects the physical properties between components. Geometrical information mainly reflects the characteristics

three-dimensional space, and effectively integrates related information of life cycle. In the process of BIM creation, it is necessary to consider the aspects of virtual construction, function simulation, performance analysis and techno-economic calculation in order to achieve the model and to ensure the effective development of the follow-up work. Using this method has many advantages. It not only can effectively coordinate the relationship, but also can further control the whole process of simulation control, so that the existing problems can be found in time to take measures to adjust them, which can improve the design efficiency and meet the relevant requirements.

\subsection{Energy-saving Analysis is More Timely and Comprehensive}

BIM is an integrated process, which mainly uses digital methods to perform various functions before construction. Because the information used is consistent throughout the process, it is necessary to observe the overall shape and performance of the whole process by related personnel before carrying out the construction, and to fully understand the features so as to ensure the follow-up work. For example, in the establishment of the BIM basic model, building energy consumption software not only can effectively carry out the analysis of energy consumption, but also can help designers do a good job of energy-saving control to achieve energy-saving purposes.

\section{Application of Green} Architecture Design based on BIM

\begin{abstract}
With the development of society, applying green technology in the construction industry has become the main development trend. In the green building, that the simulation method is used to carry out digital analysis on the energy-saving effects quickly, directly and accurately is also the main object of research at present. On the current situation, the application of BIM technology in green building design not only can effectively solve the existing problems, but also can provide more efficient technology to reduce environment pollution, improve the overall efficiency and promote the development of green building construction, so it is necessary to further strengthen the research. Based on this, this paper analyzes the green architectural design application of BIM technology.
\end{abstract}

Key words: BIM technology; Green building design; Application

Published online: $30^{\text {th }}$ Nov, 2017 


\section{Technology}

3.1 Achieve the Goal of Energy Conservation and Energy Use

The application process of BIM is mainly to use some methods to analyze all aspects of architectural design, including parameters and structures, so as to form a whole and effectively control construction quality, schedule, cost and other aspects. In the current situation, the process will require a lot of data, and BIM can effectively carry out green analysis and better protect the accuracy of data. At the same time, it is necessary to ensure the performance of building envelope structure to meet relevant reference standards. For the reference of the building structure and the actual design of the structure, it is necessary to ensure that they are in the same environment so as to effectively calculate the energy consumption. So in the application process, it is necessary to adjust each performance reasonably and control parameters control to achieve energy-saving purposes. The maximum advantage of the BIM software can be used to calculate the performance of each parameter quickly, which can further improve the work efficiency of the people. Meanwhile, it can accelerate the analog output of the data and improve the precision to achieve the goal of energy saving. In the BIM application, it can effectively carry out the layout of the building and the adjustment of the direction to ensure that the living environment can achieve the purpose of winter warm and summer cool.

\subsection{Saving Materials and Materials Utilization}

In view of the current situation, the impact of building materials on the nature is very large. Its layout destroys land, and at the same time, the energy consumption is also very large, which will directly pollute the environment and affect the survival of non-renewable resources. In view of the current situation, a lot of materials in construction projects are non-renewable natural mineral raw materials and only very few are from industrial solid waste, which will increase the consumption of energy and have an impact on environment. With the rise of green building, a large number of renewable materials can be used in the construction, which not only can reduce the environmental pollution, but also can achieve the purpose of energy saving. In the selection of materials, it is necessary to ensure that renewable, recyclable materials reach more than $10 \%$ of the total material ratio. What's more, the unit also needs to list the details and focus on the detailed records of recyclable materials. Through the BIM technology software model, the listed list is integrated, which can quickly and accurately calculate the type and weight of the materials and also can be used to determine the energy efficiency of the materials to achieve the purpose of energy saving.

\subsection{Improve the Quality of Indoor Environment}

Today, with the rapid development of society, people's living standards continue to improve and requirements for environment become higher. In the indoor environment of buildings, people's health index can be fully reflected. Under normal circumstances, the indoor environment quality will be affected by many factors, including indoor temperature and humidity, fresh air volume, the production and discharge of harmful gases, indoor airflow and outdoor wind speed and indoor objects to the release of air pollution, etc.. The use of BIM technology software model can effectively carry out the analysis of lighting, sunshine and so on to better ensure the quality of indoor environment.In the green building design, more attention will be paid to natural ventilation. It can further shorten the operating time of air-conditioning equipment, and achieve the purpose of energy-saving control. So in practical applications, it is necessary to fully grasp the location of the building and the direction of the wind and carry out design feasibly. The use of BIM software plays a very important role. It can simulate the local wind direction and wind speed, and reasonably adjust the ventilation position and size. At present, it mainly aims at the direction of doors and windows and the size of the window, etc. It also can reasonably adjust the vents layout to provide guarantee for the air circulation, such as "draughty" to ensure that indoor air flow organization can be effectively ventilated to improve indoor environment.

In short, with the development of society, green building construction has become the main trend of development. In the current situation, BIM technology is very important in the design and construction of green buildings, which can provide a new idea for the efficient design of green buildings, and meanwhile continuously promote the development of BIM technology, so it is necessary to further strengthen the research on it.

\section{References}

[1]Jia Shuang, Shen Xiaowei, Zhang Baogang. Application analysis of green building design based on BIM technology [J]. Shanxi Construction, 2016, 10:209-210.

[2] Lu Wanmei. Research on BIM technology and its application in construction design.[D]. Tianjin University, 2014.

[3]Sun Chenjunyan, Zhou Gen, Ge Yujia, Liu Yong. Research on application of BIM technology in sustainable life cycle of green building.[J]. Project Management Technology , 2017 , 02:65-69. 